\title{
¿ (2) \\ Expression of FAP in Lung Cancer and Its Correlation With the Tumor Glucose Metabolism and the Histopathology
}

\section{Xiaohui Chen}

Southern Medical University First Affiliated Hospital: Southern Medical University Nanfang Hospital

\section{Xinran Liu}

Southern Medical University First Affiliated Hospital: Southern Medical University Nanfang Hospital

\section{Lijuan Wang}

Southern Medical University First Affiliated Hospital: Southern Medical University Nanfang Hospital

\section{Wenlan Zhou}

Southern Medical University First Affiliated Hospital: Southern Medical University Nanfang Hospital

\section{Yin Zhang}

Southern Medical University First Affiliated Hospital: Southern Medical University Nanfang Hospital

\section{Ying Tian}

Southern Medical University First Affiliated Hospital: Southern Medical University Nanfang Hospital

Jianer Tan

Southern Medical University First Affiliated Hospital: Southern Medical University Nanfang Hospital

\section{Ye Dong}

Southern Medical University First Affiliated Hospital: Southern Medical University Nanfang Hospital

\section{Lilan Fu}

Southern Medical University First Affiliated Hospital: Southern Medical University Nanfang Hospital Hu bing Wu ( $\nabla$ wuhbym@163.com )

Southern Medical University First Affiliated Hospital: Southern Medical University Nanfang Hospital

\section{Research Article}

Keywords: lung cancer, fibroblast activation protein, histopathology, glucose metabolism, [18F] F-FDG $\mathrm{PET} / \mathrm{CT}$

Posted Date: January 3rd, 2022

DOI: https://doi.org/10.21203/rs.3.rs-1203456/v1

License: (c) (1) This work is licensed under a Creative Commons Attribution 4.0 International License. Read Full License 


\section{Abstract}

Purpose To explore the expression of fibroblast activation protein (FAP) in lung cancer (LC) and its correlation with tumor glucose metabolism and histopathology.

Methods From June 2018 to November 2020, a total of 73 patients with newly diagnosed LC were included. Immunohistochemical staining was used to quantify the FAP expression in the tumors. The histopathological type and tumor grade were determined on the histopathological examination. Tumor glucose metabolic parameters and tumor maximal diameter were measured on $\left[{ }^{18} \mathrm{~F}\right] \mathrm{F}$-FDG PET/CT. Univariate and multivariate analysis were used to study the correlation between FAP expression level and glucose metabolic variables and various histopathology.

Results Positive FAP expression was observed in 97.3\% (71/73) of LC lesions, which was significantly higher than $87.7 \%(64 / 73)$ of $\left[{ }^{18} \mathrm{~F}\right] \mathrm{F}-\mathrm{FDG}$ positivity on PET/CT $\left(\chi^{2}=4.818, P=0.028\right)$. In 12 early adenocarcinomas (ADCs), only 3 lesions (25\%) were positive on PET/CT, however, 10 lesions (83.3\%) were positive with FAP expression. When FAP expression was classified to low level (scores $\leq 3$ ) and high level (scores>4), high FAP level was found in $80.8 \%$ tumors and low FAP level in other $19.2 \%$ tumors. High FAP level was identified in $100.0 \%$ of squamous cell carcinoma (SCC), $85.7 \%$ of ADCs, $66.7 \%(4 / 6)$ of large cell neuroendocrine carcinoma (LCNC) and 40.0\% (4/10) of small cell lung cancer (SCLC) $(P<0.05)$. In the non-mucinous ADC lesions, on univariate analysis, FAP expression level showed close relationships with tumor metabolism parameters (SUVmax, SUVmean, and TLG), tumor diameter, tumor grade and lesion attenuation $(P<0.05)$.

Conclusion The present study demonstrates that FAP expresses widely in LC and has a great variant level in different histopathological types. High positive rate of FAP expression implies FAP targeted imaging may be a sensitive modality for diagnosing LC, especially for early ADCs, and may serve as an alternative of $\left[{ }^{18} \mathrm{~F}\right]$ F-FDG PET/CT.

\section{Introduction}

Lung Cancer (LC) is the leading cause of cancer-related deaths in the world ${ }^{[1]}$. Despite recent developments in the diagnosis, classification and therapy, the overall survival is still poor ${ }^{[2]}$. Unfortunately, most patients present with advanced stage LC at diagnosis, mainly because of lack of disease-specific symptoms ${ }^{[3]}$. More and more studies have shown that the occurrence and progression of tumors are the result of dynamic interactions between tumor cells and various components in the tumor microenvironment (TME) ${ }^{[4]}$. Therefore, TME emerges to be a promising target for diagnosis and therapy for LC, which is hoped to play a role in tumor early detection and improving the current unfavorable treatment effect.

Fibroblast activation protein (FAP), a type $₫$ membrane-bound glycoprotein belonging to the dipeptidyl peptidase 4 family, is an imperative factor in cancer-associated fibroblasts (CAFs), which is a 
fundamental component of the $\mathrm{TME}^{[5]}$. This protein is observed to be usually highly upregulated during tissue remodeling events, including tumorigenesis ${ }^{[6,7]}$ and is relevant to tumor growth, migration, and progression via the secretion of cytokines and chemokines ${ }^{[8,9]}$. Currently, FAP-targeting radioligands have been developed for PET/CT imaging and targeted radionuclide therapy for a variety of cancer including LC, colorectal cancer (CRC) and so on ${ }^{[10,11]}$, which may produce a significant impact on the diagnosis and treatment of LC in the future.

It is well known that LC contains a group of heterogeneous tumors, with several histopathological types including adenocarcinoma (ADC), squamous cell carcinoma (SCC), small cell lung cancer (SCLC), large cell neuroendocrine carcinoma(LCNC) and so on ${ }^{[1]}$.This disease shows great variations in biological behavior in different histopathological types and different stages ${ }^{[2]}$.It is postulated that FAP, as a fundamental factor of the TME, may have some relationship to the histopathology and the disease stages in LC.

$\left[{ }^{18} \mathrm{~F}\right]$ F-FDG PET/CT is a functional imaging modality, which plays an important role in the diagnosis, staging and treatment response evaluation in variety of tumors ${ }^{[12,13]}$. $\left[{ }^{18} \mathrm{~F}\right] \mathrm{F}-\mathrm{FDG}$ PET/CT can be used to quantify the metabolism of the glucose in the cancer cells and the TME in the tumor, which is usually positively related to the aggressiveness and prognosis ${ }^{[14,15]}$. A tumor with highly aggressiveness always presents with high glucose metabolism. It is also postulated that FAP may have some relationship to tumor glucose metabolism.

In the present study, we performed an immunohistochemical analysis to measure the expression of FAP in the primary tumors of LC in 73 patients, who simultaneously received the $\left[{ }^{18} \mathrm{~F}\right] \mathrm{F}-\mathrm{FDG}$ PET/CT recently. Furthermore, we also investigated the relationships of the expression level of FAP with tumor histopathological types and other clinical indicators. This study may contribute to a better understanding of the biological role of FAP in LC and could be useful to further guide clinical translation of novel FAPtargeting PET/CT imaging and therapy.

\section{Materials And Methods}

\section{Patient Cohort}

The Institutional Review Board of Nanfang Hospital at Southern Medical University approved the present retrospective study and waived the requirement for written informed consent because of the retrospective nature of the study.

From June 2018 to November 2020, a total of 73 patients with newly diagnosed LC who underwent $\left[{ }^{18} \mathrm{~F}\right]$ F-FDG PET/CT at Nanfang Hospital for staging before the surgery or other anti-tumor therapy were included. The inclusion criteria were as follows: (1) Final diagnosis of LC was established by histopathological examination; (2) No neo-adjuvant therapy was administered before the PET/CT scans; and (3) FAP immunohistochemical staining was available. The expression of FAP was measured using 
the immunohistochemical staining. The clinical information was collected through Picture Archiving and Communication Systems (PACS). Glucose metabolic parameters including the maximal standard uptake value (SUVmax), mean standard uptake value (SUVmean), metabolic tumor volume (MTV) and total lesion glycolysis (TLG), and tumor diameter were measured on $\left[{ }^{18} \mathrm{~F}\right]$ F-FDG PET/CT.

\section{$\left[{ }^{18} \mathrm{~F}\right]$ F-FDG PET/CT Scans}

$\left[{ }^{18} \mathrm{~F}\right]$ F-FDG PET/CT scans were performed using a Biograph mCTx scanner (Siemens Healthcare, Germany) or a total-body PET/CT scanner (UEXPLORER, United Imaging Healthcare, China). The patients were instructed to fast for at least 4-6 h, and their blood glucose levels were monitored with a glucometer to ensure the levels below $10.0 \mathrm{mmol} / \mathrm{L}$ prior to $\left[{ }^{18} \mathrm{~F}\right] \mathrm{F}$-FDG injection. $\left[{ }^{18} \mathrm{~F}\right] \mathrm{F}-\mathrm{FDG}$ was manufactured using a tracer synthesis system (TRACERlab FX $\mathrm{FDG}_{\text {; }}$ GE Healthcare, United States) and had a >95\% radiochemical purity. Approximately $60 \mathrm{~min}$ after the intravenous injection of $318-524 \mathrm{MBq}(8.6-14.2 \mathrm{mCi}$, $150 \mu \mathrm{Ci} / \mathrm{kg})\left[{ }^{18} \mathrm{~F}\right] \mathrm{F}-\mathrm{FDG}, \mathrm{PET} / \mathrm{CT}$ was performed with a 5 -minute single bed position three-dimensional acquisition for UEXPLORER scanner and $5-6$ bed positions three-dimensional acquisition with $2 \mathrm{~min} / \mathrm{bed}$ position for Biograph mCTx scanner according to established protocols in our center ${ }^{[16,17]}$.

The acquired PET and CT images were registered and analyzed using the Syngo MI workplace (Siemens Healthcare, Germany) or Image Workstation (MedEx (Beijing) Technology Limited Corporation, China). All the PET/CT images were independently read by two certified nuclear medicine physicians with over five years of experience. Both physicians were blinded to the clinical and histopathological information and the results of FAP expression. According to the attenuation of lesions, the ADC lesions were classified into pure ground glass nodules (pGGN), mixed ground glass nodules (mGGN) and solid nodules (SN). For the semiquantitative analysis, a region of interest (ROI) was drawn along the margin of the lesion on the axial PET image and automatically adapted to a three-dimensional volume-of-interest at a $60 \%$ isocontour, which was used to measure the SUVmax, SUVmean, MTV and TLG. TLG was calculated using the formula: TLG = SUVmean×MTV.

\section{Histologic Diagnosis}

Resected or biopsied pathologic lung specimens (hematoxylin and eosin- stained slides) were independently reviewed by two certified pathologists to determine the histopathological type (ADC, SCC, SCLC, LCNC and so on) and ADC subtype. The malignant grade of non-mucinous ADC subtype was partitioned based on the IASLC/ATS/ERS criteria ${ }^{[18]}$. According to the literature ${ }^{[19]}$, patients with nonmucinous ADC were classified into three subgroups: low grade group including adenocarcinoma in situ (AIS), minimally invasive adenocarcinoma (MIA) and lepidic predominant invasive adenocarcinoma(LPA), medium grade group including acinar predominant invasive adenocarcinoma (APA), papillary predominant invasive adenocarcinoma (PPA) and high grade group including solid predominant invasive adenocarcinoma(SPA) and micropapillary predominant invasive adenocarcinoma (MPA). The pathologists were blinded with the results of $\left[{ }^{18} \mathrm{~F}\right]$ F-FDG PET/CT, the results of FAP expression and other clinical information. 


\section{Immunohistochemical Analysis}

LC tissue samples were acquired via biopsy or surgical resection, including 11 biopsy samples and 60 surgical resection samples. Paraffin-embedded tissue sections were deparaffinized with xylene and rehydrated in a graded series of ethanol solutions. Antigen retrieval was performed by heating the slides twice in $0.01 \mathrm{~mol} / \mathrm{L}$ sodium citrate buffer, $\mathrm{pH} 6.0$, in a microwave oven (13 min, $850 \mathrm{~W}$ ). Endogenous peroxidase was then blocked with $0.3 \% \mathrm{H}_{2} \mathrm{O}_{2}$ in methanol for $15 \mathrm{~min}$ at room temperature.

Immunohistochemical staining was performed by incubating the slides with a rabbit anti-FAP antibody (\#66562S, Cell Signaling Technology, United States) at a dilution of 1:100 at $4{ }^{\circ}$ Covernight. Serial sections were stained with a horseradish peroxidase enzyme-labelled polymer conjugated to antimouse/rabbit immunoglobulins, according to the instructions of the Chemmate EnVision/ Mo\&Rb Detection Kit (GK500705, Gene Tech Company Limited, Shanghai, China).

The staining of FAP in tumor tissue was calculated based on the percent positivity of stained cells and the staining intensity. The percent positivity was scored as 0 (absent or $<1 \%), 1(1 \%-10 \%), 2(11 \%-50 \%)$, or $3(>50 \%)^{[20]}$. Five to ten regions of interest were manually selected and each selected region was individually calculated for percent of positive cells. The average percentage of positive cells for all regions was calculated as mean for statistical comparisons. The staining intensity was scored as 0 (no staining), 1 (weak staining), 2 (moderate staining), or 3 (strong staining). The percent positivity and staining intensity were determined in a double-blinded manner. When the score of a case did not coincide, both pathologists came to an agreement after a discussion while viewing specimens under a doubleheaded microscope. The FAP expression score based on immunostaining was calculated as percent positivity score plus staining intensity score and ranged from 0 to 6 . The FAP expression level was defined as - $0 ; 1^{+}, 1-3 ; 2^{+}, 4-5$; or $3^{+}, 6$.

\section{Statistical Analysis}

All statistical analyses were performed using SPSS version 25.0 (IBM Corporation, Armonk, NY, USA). The classification performance of clinicopathological factors and metabolic parameters between high FAP expression group and low FAP expression group in the tumor were analyzed. Quantitative data that were normally distributed are expressed as mean \pm standard deviation, and the independent sample t-test was used for comparison between the two groups. Quantitative data were not normally distributed are expressed as median (interquartile range), and the Mann-Whitney test was used for comparison between the two groups. Qualitative data are expressed as number and percentage ( $[\%])$, and the chi-squared test or Fisher's exact probability method were used for comparison between two groups. Multiple logistic regression analyses were used to analyze the association between FAP expression and $\left[{ }^{18} \mathrm{~F}\right] \mathrm{F}$-FDG uptake, histopathological diagnosis, and other clinical parameters. A p-value $<0.05$ indicated statistical significance.

\section{Results}




\section{Patient Characteristics}

Of the 73 included patients, 49 (67.1\%) were male and 24 (32.9\%) were female. Median age was 60.5 years (range: $42-79$ years). ADC was diagnosed in 42 (57.5\%) patients (2 mucinous and 40 non-mucinous ADCs), SCC in 15 (20.6\%) patients, SCLC in 10(13.7\%) patients, and LCNC in 6 (8.2\%) (Table 1, 2). The

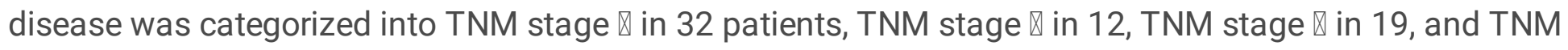

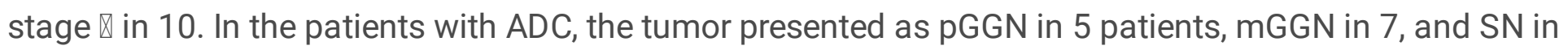
30 (Table 3). Low grade ADC was found in 12 patients, medium grade ADC in 16, and high grade ADC in 12 (Table 3). 
Table 1

Patient Characteristics

\begin{tabular}{|ll|}
\hline Variable & N(\%) or $\mathbf{X} \pm$ SD \\
\hline Gender & \\
\hline Male & $49(67.1)$ \\
\hline Female & $24(32.9)$ \\
\hline Age(yr) & \\
\hline$<60$ & $37(50.7)$ \\
\hline$\geq 60$ & $36(49.3)$ \\
\hline Smoking history & \\
\hline Never smoker & $32(43.8)$ \\
\hline Smoker & $41(56.2)$ \\
\hline TNM stage & \\
\hline$\square$ & $32(43.8)$ \\
\hline$\square$ & $12(16.5)$ \\
\hline$\square$ & $19(26.0)$ \\
\hline$\square$ & $10(13.7)$ \\
\hline Histopathological type & \\
\hline SCLC & $10(13.7)$ \\
\hline LCLC & $6(8.2)$ \\
\hline ADC & $42(57.5)$ \\
\hline SCC & $15(20.5)$ \\
\hline TMD (cm) & $3.08 \pm 1.67$ \\
\hline SUVmax & $12.88 \pm 8.56$ \\
\hline SUVmean & $7.76 \pm 5.41$ \\
\hline MTV (cm ${ }^{3}$ ) & $10.17 \pm 16.47$ \\
\hline TLG & \\
\hline
\end{tabular}


Table 2

Univariate analysis of the variables related to FAP expression in lung cancer $(n=73)$

\begin{tabular}{|c|c|c|c|}
\hline \multirow[t]{2}{*}{ Variable } & \multicolumn{2}{|c|}{ FAP expression in the tumor, $n(\%)$} & \multirow[t]{2}{*}{$P$ value } \\
\hline & Low & High & \\
\hline Gender & & & 0.703 \\
\hline Male & $10(20.4)$ & $39(79.6)$ & \\
\hline Female & $4(16.7)$ & $20(83.3)$ & \\
\hline Age(yr) & & & 0.591 \\
\hline$<60$ & $8(21.6)$ & $29(78.4)$ & \\
\hline$\geq 60$ & $6(16.7)$ & $30(83.3)$ & \\
\hline Smoking history & & & 0.496 \\
\hline Never smoker & $5(15.6)$ & $27(84.4)$ & \\
\hline Smoker & $9(22.0)$ & $32(78.0)$ & \\
\hline TNM stage & & & 0.533 \\
\hline ૧ & $8(25.0)$ & $24(75.0)$ & \\
\hline ૧ & $1(8.3)$ & 11(91.7) & \\
\hline प & $4(21.1)$ & 15(78.9) & \\
\hline प & $1(10.0)$ & $9(90.0)$ & \\
\hline Histopathological type & & & 0.001 \\
\hline SCLC & $6(60.0)$ & $4(40.0)$ & \\
\hline LCLC & 2(33.3) & $4(66.7)$ & \\
\hline ADC & $6(14.3)$ & $36(85.7)$ & \\
\hline SCC & $0(0.0)$ & 15(100.0) & \\
\hline TMD (cm) & $2.65 \pm 1.80$ & $3.18 \pm 1.63$ & 0.126 \\
\hline SUVmax & $13.3 \pm 14.50$ & $12.77 \pm 6.62$ & 0.420 \\
\hline SUVmean & $8.25 \pm 9.50$ & $7.63 \pm 4.00$ & 0.458 \\
\hline $\operatorname{MTV}\left(\mathrm{cm}^{3}\right)$ & $6.78 \pm 9.90$ & $10.97 \pm 17.65$ & 0.348 \\
\hline TLG & $89.00 \pm 159.54$ & $102.64 \pm 219.71$ & 0.297 \\
\hline
\end{tabular}


Table 3

Univariate analysis of the variables related to FAP expression in nonmucinous lung adenocarcinoma ( $\mathrm{n}-40)$

\begin{tabular}{|c|c|c|c|}
\hline \multirow[t]{2}{*}{ Variable } & \multicolumn{2}{|c|}{ FAP expression in the tumor, $n(\%)$} & \multirow[t]{2}{*}{$P$ value } \\
\hline & Low & High & \\
\hline Gender & & & 1.000 \\
\hline Male & $3(15.0)$ & 17(85.0) & \\
\hline Female & $3(15.0)$ & 17(85.0) & \\
\hline Age(yr) & & & 0.451 \\
\hline$<60$ & $4(19.0)$ & 17(81.0) & \\
\hline$\geq 60$ & $2(10.5)$ & 17(89.5) & \\
\hline \multicolumn{4}{|l|}{ Smoking history } \\
\hline Never smoker & $4(16.0)$ & $21(84.0)$ & 0.819 \\
\hline Smoker & $2(13.3)$ & 13(86.7) & \\
\hline TNM stage & & & 0.195 \\
\hline$\square$ & $6(25.0)$ & 18(75.0) & \\
\hline ૧ & $0(0.0)$ & $5(100.0)$ & \\
\hline$\square$ & $0(0.0)$ & $7(100.0)$ & \\
\hline$\square$ & $0(0.0)$ & $4(100.0)$ & \\
\hline Lesion attenuation & & & 0.004 \\
\hline pGGO & $1(25.0)$ & $3(75.0)$ & \\
\hline mGGO & $4(50.0)$ & $4(50.0)$ & \\
\hline SN & 1(3.6) & $27(96.4)$ & \\
\hline Tumor grade & & & 0.008 \\
\hline A: AIS + MIA + LPA & $5(41.7)$ & $7(58.3)$ & \\
\hline B: APA + PPA & $1(6.3)$ & 15(93.7) & \\
\hline C: SPA + MPA & $0(0.0)$ & 12(100.0) & \\
\hline TMD (cm) & $1.60 \pm 1.10$ & $2.50 \pm 1.60$ & 0.028 \\
\hline SUVmax & $1.90 \pm 2.60$ & $10.60 \pm 11.10$ & 0.004 \\
\hline SUVmean & $1.15 \pm 1.60$ & $6.40 \pm 6.50$ & 0.004 \\
\hline
\end{tabular}




\begin{tabular}{|llll|}
\hline Variable & \multicolumn{2}{l}{ FAP expression in the tumor, $\boldsymbol{n}(\%)$} & P value \\
\cline { 2 - 3 } & Low & High & \\
\hline MTV $\left(\mathrm{cm}^{3}\right)$ & $1.77 \pm 1.30$ & $3.96 \pm 6.50$ & 0.058 \\
\hline TLG & $2.22 \pm 4.80$ & $22.10 \pm 41.5$ & 0.004 \\
\hline
\end{tabular}

\section{FAP Expression in LC lesions}

Of the total of $73 \mathrm{LCs}$, the expression of FAP in the tumor was positive in $97.3 \%$ (71/73) of patients, except two patients with ADC. When FAP expression was classified to low level (scores $\leq 3$ ) and high level (scores $>4$ ), high FAP level was identified in $80.8 \%$ tumors and low FAP level in other $19.2 \%$ tumors (Figure 1, 2). Different histopathologic types of LCs showed variant FAP expression levels. High FAP level was found in $100 \%$ (15/15) lesions of SCCs, $85.7 \%$ (36/42) of ADCs, $66.7 \%(4 / 6)$ of LCNCs and $40 \%(4 / 10)$ of SCLCs, respectively (Table 2) (Figure 3). SCC and ADC had significantly more lesions with high FAP level than that of SCLC $(P=0.001$ and $P=0.002)$ and SCC also had more lesions with high FAP level than that of LCNC $(P=0.019)$. There were no significant differences in the number of tumors with high FAP level between SCC and ADC, or ADC and LCNC, or ADC and SCLC, or LCNC and SCLC, respectively ( $P>0.05)$. Similarly, higher FAP score was found in SCC and ADC, compared to those of LCNC and SCLC $(P<0.01)$ (Table 4).

Table 4

FAP expression and tumor glucose metabolic parameters in 73 patients with lung cancer

\begin{tabular}{|c|c|c|c|c|c|c|}
\hline \multirow[t]{2}{*}{ Variable } & \multicolumn{2}{|c|}{ FAP expression } & \multicolumn{4}{|c|}{${ }^{18} \mathrm{~F}$-FDG PET/CT } \\
\hline & $\begin{array}{l}\text { Positivity } \\
\text { (\%) }\end{array}$ & Score & $\begin{array}{l}\text { Positivity } \\
(\%)\end{array}$ & SUVmax & MTV & TLG \\
\hline SCC & $\begin{array}{l}100.0 \% \\
(15 / 15)\end{array}$ & $5.53 \pm 0.64$ & $\begin{array}{l}100.0 \% \\
(15 / 15)\end{array}$ & $17.70 \pm 4.43$ & $14.48 \pm 11.89$ & $145.55 \pm 120.64$ \\
\hline ADC & $\begin{array}{l}95.2 \% \\
(40 / 42)\end{array}$ & $4.88 \pm 1.60$ & $\begin{array}{l}78.6 \% \\
(33 / 42)\end{array}$ & $9.27 \pm 6.52$ & $5.54 \pm 7.17$ & $35.03 \pm 50.98$ \\
\hline LCLC & $\begin{array}{l}100.0 \% \\
(6 / 6)\end{array}$ & $4.17 \pm 1.60$ & $\begin{array}{l}100.0 \% \\
(6 / 6)\end{array}$ & $19.42 \pm 7.77$ & $32.27 \pm 45.24$ & $427.19 \pm 604.21$ \\
\hline SCLC & $\begin{array}{l}100.0 \% \\
(10 / 10)\end{array}$ & $3.20 \pm 1.13$ & $\begin{array}{l}100.0 \% \\
(10 / 10)\end{array}$ & $16.84 \pm 13.55$ & $9.87 \pm 11.11$ & $108.40 \pm 127.30$ \\
\hline$P$ value & 0.678 & 0.000 & 0.056 & 0.000 & 0.025 & 0.000 \\
\hline
\end{tabular}

\section{The Tumor Glucose Metabolism}


In 73 LC patients included, high level of $\left[{ }^{18} \mathrm{~F}\right] \mathrm{F}-\mathrm{FDG}$ uptake (SUVmax $>2.5$ ) in the tumors were found in $87.7 \%(64 / 73)$ patients. Low level of $\left[{ }^{18} \mathrm{~F}\right] \mathrm{F}$-FDG uptake $(0<\mathrm{SUVmax}<2.5)$ in the tumors were observed in $5 / 8$ mGGO lesions. No $\left[{ }^{18} \mathrm{~F}\right]$ F-FDG uptake (SUVmax $\left.=0\right)$ in the tumors were found in 4/4 pGGO lesions. The mean SUVmax of the primary tumors was 12.9 (range $=0.0-54.1$ ), mean SUVmean was 7.8 (range $=$ 0.0-36.7), MTV was 10.2 (range $=0.0-119.4$ ) and TLG was 100.0 (range $=0.0-1585.6$ ), respectively. When SUVmax $>2.5$ was considered positive, $\left[{ }^{18} \mathrm{~F}\right] \mathrm{F}$-FDG PET/CT showed $87.7 \%$ positivity in the LC lesions, which was significantly lower than that $(97.3 \%)$ of positive expression of FAP $(P=0.028)$. Positive $\left[{ }^{18} F\right] F-$ FDG PET/CT was observed in 100\% (15/15) of SCC tumors, $78.5 \%$ (33/42) of ADC tumors, $100 \%(6 / 6)$ of LCNC tumors and $100 \%(10 / 10)$ of SCLC tumors, respectively. The glucose metabolic parameters of SCC, ADC, LCNC and SLCL lesions were listed in Table 4. ADCs had a large variant level of $\left[{ }^{18} \mathrm{~F}\right] \mathrm{F}-\mathrm{FDG}$ uptake and showed a lower mean SUVmax, MTV, TLG and positive rate on PET/CT compared to other histopathologic LCs (Table 4). In ADC tumors, coincident [ $\left.{ }^{18} \mathrm{~F}\right]$ F-FDG uptake and FAP expression were observed in the SN tumors, but inconsistent $\left[{ }^{18} \mathrm{~F}\right] \mathrm{F}$-FDG uptake and FAP expression were commonly found in the pGGO and mGGO tumors (Figure 4). In 12 early ADCs, which presented as pGGO or mGGO on CT, positive $\left[{ }^{18} \mathrm{~F}\right]$ F-FDG PET/CT was found in only 3 lesions (25\%), which was significantly lower than 83.3\% (10/12) of positive FAP expression on immunohistochemical staining ( $P=0.004)$ (Figure 4).

\section{Association of FAP Expression Level and Tumor Glucose and Clinicopathological Variables}

When all the patients were included to analyze the association between FAP expression level and tumor glucose metabolic parameters or clinicopathological variables, univariate analysis showed only the histopathology of the tumor had a close relationship with the FAP expression level $(P<0.05)$. Neither the semiquantitative parameters of glucose metabolism, including SUVmax, SUVmean, MTV and TLG, nor the tumor maximal diameter, stages, age, gender and smoking history, had close relationship with the FAP expression level $(P>0.05)$ (Table 2).

However, when only non-mucinous ADCs were included to analyze the association between FAP expression level and various variables, univariate analysis showed that SUVmax, SUVmean and TLG were significantly related to FAP level $(P<0.01)$, but not MTV $(P>0.05)$ (Figure 5$)$. The tumors with high FAP level had significantly higher levels of SUVmax, SUVmean and TLG than those of the tumor with low FAP level $(P<0.01)$ (Table 3). Meanwhile, the tumor maximal diameter, tumor grade and tumor attenuation were also found to be closely associated with FAP level in the tumor $(P<0.05)$ (Figure 5$)$. The tumors with high FAP level tended to have significantly larger tumor size, more solid attenuation on $C T$ and higher grade on histopathology than those of the tumors with low FAP level $(P<0.05)$ (Table 3$)$.Nevertheless, FAP level showed no significant relationship with the age, sex, smoking history and TNM stage $(P>0.05)$ (Table 3).Multivariate analysis was then used to analyze the association between FAP level and the above six related factors on the univariate analysis (three glucose metabolic parameters, tumor maximal diameter, tumor grade and lesion attenuation). However, it revealed that none of above 6 factors had significant association with the expression level of FAP in the non-mucinous ADC tumors. 


\section{Discussion}

It has long been recognized that cancer-associated fibroblasts (CAFs) play an important role in tumor growth-promoting, rendering cells to invade surrounding tissues through cell-to-cell interactions and secretion of different invasive molecules, as well as producing angiogenic factors for the formation of new blood vessels, all of which contribute to disease progression ${ }^{[21]}$. FAP, as an important surface marker of CAFs, has attracted more and more attention, especially after the radiopharmaceuticals targeted to FAP were recently successfully developed for tumors imaging and showed a promising prospect for treating malignant tumors ${ }^{[10,22,23]}$. LC may be one of the optimum tumors for FAP-targeted imaging and therapy because it was found to be one of 6 cancers presented with the highest uptake level (SUVmax $>12$ ) of $\left[{ }^{68} \mathrm{Ga}\right]$ Ga-FAPI-04, a novel FAP targeting PET tracer ${ }^{[10,11]}$. However, so far, FAPI PET/CT imaging has mainly been performed in patients with advanced LC ${ }^{[11,24,25]}$. It will be of great significance to uncover whether this novel PET tracer is sensitive in diagnosing LC and which histopathological type of LC can be more beneficial from the FAPI targeted therapy. For a targeted imaging or therapy, the diagnostic and therapeutic effects largely depend on the expression of the target. Therefore, a full understanding of the expression of FAP in LC helps to guide the clinical translation of FAP-targeted imaging and therapy for this disease. Nevertheless, few papers have described the whole picture of the expression of FAP in LC.

In the present study, clinical samples from the patients with LC were analyzed for the expression of FAP using immunohistochemistry staining. It was observed in the present study that nearly all (97.3\%) of the LCs had positive expression of FAP, which was significantly higher than $87.7 \%$ positive rate of $\left[{ }^{18} \mathrm{~F}\right] \mathrm{F}-\mathrm{FDG}$ PET/CT for LC, indicating that FAP-targeted PET/CT may be a more sensitive modality for diagnosing LC. $\left[{ }^{18} \mathrm{~F}\right] \mathrm{F}-\mathrm{FDG} \mathrm{PET} / \mathrm{CT}$ is a standard imaging modality recommended for diagnosing and staging LC by NCCN guidance ${ }^{[26,27]}$. However, it is not sensitive for the detection of the low invasive tumors, such as those presented as pGGO or mGGO on CT, which usually are non-FDG avid ${ }^{[28]}$. In the present study, only 3 lesions of 12 early $A D C s$, which presented as $\mathrm{PGGO}$ and $\mathrm{mGGO}$ lesions on $\mathrm{CT}$, were observed to be $\left[{ }^{18} \mathrm{~F}\right] \mathrm{F}$ FDG positivity on PET/CT. On the contrary, 10 of them showed positive expression of FAP, which implied that FAP-targeted PET/CT may be a supplement to $\left[{ }^{18} \mathrm{~F}\right]$ F-FDG PET/CT in diagnosing the early ADCs. A case report confirmed the potential advantage of FAPI PET/CT in imaging early LC, which reported $\left[{ }^{68} \mathrm{Ga}\right]$ Ga-FAPI-04 was highly taken by an early LC, presented as $\mathrm{mGGO}$ on CT, while no uptake of $\left[{ }^{18} \mathrm{~F}\right] \mathrm{F}-\mathrm{FDG}$ was observed in the lesion ${ }^{[29]}$.

It was also found for the first time that there was a great variation of FAP expression level in LCs with different histopathological types. SCC was found to have the most lesions with high FAP level, followed by ADC, LCNC and SCLC. This finding may also be useful for guiding the radionuclide ( ${ }^{177}$ Lu or ${ }^{90} \mathrm{Y}$ ) labeled FAP-targeted therapy. It is well known, despite the introduction of multiple new therapy for LC, the survival rate remains relatively low ${ }^{[2]}$, so it is necessary to find more effective treatment for LC. Our data indicated that radionuclide labeled FAP-targeted therapy may be an expectable treatment for SCCs and ADCs, which showed high FAP expression level in $100 \%$ and $85.7 \%$ of the tumors. On the contrary, low 
FAP expression was found in most of the lesions of SCLC, implying radionuclide labeled FAP-targeted therapy may be not suitable for SCLC. Our result was consistent with that reported by Wei Yuchun et al, which showed lower uptake of $\left.{ }^{68} \mathrm{Ga}\right] \mathrm{Ga}-\mathrm{FAPI}-04$ in SCLCs than other types of LC ${ }^{[30]}$.

In the ADC tumors, on univariate analysis, our study revealed the FAP level had close relationships with the tumor grade, lesion diameter, lesion attenuation and glucose metabolism $(P<0.05)$. High FAP level in the tumors were often accompanied by larger tumor lesions, more solid attenuation and higher glucose metabolism in ADCs, which were reported to be positively related with high tumor aggressiveness ${ }^{[14,19]}$. Therefore, it implies that FAPI imaging may be helpful to predict tumor invasiveness. Similar result was also reported by P. Moreno-Ruiz et al ${ }^{[31]}$. However, this relationship was not identified on the multivariate analysis, so further research with larger sample size is needed to determine whether this correlation really exists.

There are some limitations to this study. First, this study was retrospective study, there may have been a certain degree of bias. Second, the included sample sizes of patients were small, especially the number of LCNC and SCLC, which may fail to reflect the whole picture of the expression of FAP in LCNC and SCLC. Lastly, no benign disease was included, which make the diagnostic specificity of FAP expression for lung cancer could not be determined.

\section{Conclusion}

The present study demonstrates that nearly all the LCs have positive expression of FAP and different histopathological types of LC have variant levels of FAP expression. This study implies that FAP-targeted imaging may be a sensitive modality for diagnosing LC, especially for early ADCs. It also implies that FAP-targeted therapy may open a new door for treating SCCs and ADCs, which always have high FAP expression level. Due to a relatively small sample size in the present study, more researches are warranted to confirm our findings.

\section{Declarations}

\section{Authors' contributions}

Conceptualization: Hubing Wu

Formal analysis and investigation: Xiaohui Chen, Xinran Liu, Lijuan Wang, Wenlan Zhou, Yin Zhang, Ying Tian, Jianer Tan, Ye Dong, Lilan Fu, Hubing Wu

Writing-original draft preparation: Xiaohui Chen, Xinran Liu, Lijuan Wang

Writing-review and editing: Hubing Wu

Funding acquisition: Hubing Wu 
Supervision: Hubing Wu

All authors read and approved the final manuscript.

\section{Funding}

This work was supported financially by the National Science Foundation of China under grants 81873905.

\section{Data availability}

All data were transparent. The data used in the current study are available from the corresponding author on reasonable request.

\section{Compliance with Ethical Standards}

\section{Conflict of interest}

The authors declare that they have no conflict of interest.

\section{Ethics approval}

Ethical approval was waived by the local Ethics Committee of Southern Medical University in view of the retrospective nature of the study.

\section{Consent to participate}

Due to the retrospective nature of the study, informed consent was not obtained in the study.

\section{References}

1. Bray, F. et al. Global cancer statistics 2018: GLOBOCAN estimates of incidence and mortality worldwide for 36 cancers in 185 countries. CA Cancer J Clin. 2018; 68: 394-424. http://doi:10.3322/caac.21492

2. de Sousa, V. M. L. \& Carvalho, L. Heterogeneity in Lung Cancer. Pathobiology. 2018; 85: 96-107. http://doi:10.1159/000487440

3. Jain, D. Lung Molecular Cytopathology: EGFR and Beyond. J Cytol. 2019; 36: 124-127. http://doi:10.4103/JOC.JOC_135_18

4. Nurmik, M., Ullmann, P., Rodriguez, F., Haan, S. \& Letellier, E. In search of definitions: Cancerassociated fibroblasts and their markers. Int J Cancer. 2020; 146: 895-905. http://doi:10.1002/ijc.32193

5. Yu, D. M. et al. The dipeptidyl peptidase IV family in cancer and cell biology. FEBS J. 2010; 277: 1126-1144. http://doi:10.1111/j.1742-4658.2009.07526.x 
6. Chen, L., Qiu, X., Wang, X. \& He, J. FAP positive fibroblasts induce immune checkpoint blockade resistance in colorectal cancer via promoting immunosuppression. Biochem Biophys Res Commun. 2017; 487: 8-14. http://doi:10.1016/j.bbrc.2017.03.039

7. Mankaney, G. et al. Gastric cancer in FAP: a concerning rise in incidence. Fam Cancer. 2017; 16: 371376. http://doi:10.1007/s10689-017-9971-3

8. Bhowmick, N. A., Neilson, E. G. \& Moses, H. L. Stromal fibroblasts in cancer initiation and progression. Nature. 2004; 432: 332-337. http://doi:10.1038/nature03096

9. Hamson, E. J., Keane, F. M., Tholen, S., Schilling, O. \& Gorrell, M. D. Understanding fibroblast activation protein (FAP): substrates, activities, expression and targeting for cancer therapy. Proteomics Clin Appl. 2014; 8: 454-463. http://doi:10.1002/prca.201300095

10. Giesel, F. L. et al. (68)Ga-FAPI PET/CT: Biodistribution and Preliminary Dosimetry Estimate of 2 DOTA-Containing FAP-Targeting Agents in Patients with Various Cancers. J Nucl Med. 2019; 60: 386392. http://doi:10.2967/jnumed.118.215913

11. Kratochwil, C. et al. (68)Ga-FAPI PET/CT: Tracer Uptake in 28 Different Kinds of Cancer. J Nucl Med. 2019; 60: 801-805. http://doi:10.2967/jnumed.119.227967

12. Castello, A., Rossi, S. \& Lopci, E. 18F-FDG PET/CT in Restaging and Evaluation of Response to Therapy in Lung Cancer: State of the Art. Current Radiopharmaceuticals. 2020; 13: 228-237. http://doi:10.2174/1874471013666191230144821

13. Sharma, A. et al. Utility of early dynamic and delayed post-diuretic (18)F-FDG PET/CT SUVmax in predicting tumour grade and T-stage of urinary bladder carcinoma: results from a prospective single centre study. Br J Radiol. 2017; 90: 20160787. http://doi:10.1259/bjr.20160787

14. Guo, J. et al. VEGF-A and its isoform VEGF(1)(2)(1) mRNA expression measured by quantitative realtime RT-PCR: correlation with F-18 FDG uptake and aggressiveness of lung adenocarcinoma: preliminary study. Ann Nucl Med. 2011; 25: 29-36. http://doi:10.1007/s12149-010-0427-1

15. Ruilong, Z. et al. Diagnostic value of 18F-FDG-PET/CT for the evaluation of solitary pulmonary nodules: a systematic review and meta-analysis. Nucl Med Commun. 2017; 38: 67-75. http://doi:10.1097/MNM.0000000000000605

16. Badawi, R. D. et al. First Human Imaging Studies with the EXPLORER Total-Body PET Scanner. J Nucl Med. 2019; 60: 299-303. http://doi:10.2967/jnumed.119.226498

17. Ren, Y. Y. et al. Whole-body (18)F-FDG PET/CT for M staging in the patient with newly diagnosed nasopharyngeal carcinoma: Who needs? Eur J Radiol. 2017; 89: 200-207. http://doi:10.1016/j.ejrad.2017.02.002

18. Travis, W. D. et al. International association for the study of lung cancer/american thoracic society/european respiratory society international multidisciplinary classification of lung adenocarcinoma. J Thorac Oncol. 2011; 6: 244-285. http://doi:10.1097/JT0.0b013e318206a221

19. Nakamura, H. et al. Close association of IASLC/ATS/ERS lung adenocarcinoma subtypes with glucose-uptake in positron emission tomography. Lung Cancer. 2015; 87: 28-33. http://doi:10.1016/j.lungcan.2014.11.010 
20. Liao, Y., Ni, Y., He, R., Liu, W. \& Du, J. Clinical implications of fibroblast activation protein-alpha in nonsmall cell lung cancer after curative resection: a new predictor for prognosis. J Cancer Res Clin Oncol. 2013; 139: 1523-1528. http://doi:10.1007/s00432-013-1471-8

21. Koustoulidou, S. et al. Cancer-Associated Fibroblasts as Players in Cancer Development and Progression and Their Role in Targeted Radionuclide Imaging and Therapy. Cancers (Basel). 2021; 13. http://doi:10.3390/cancers13051100

22. Fischer, E. et al. Radioimmunotherapy of fibroblast activation protein positive tumors by rapidly internalizing antibodies. Clin Cancer Res. 2012; 18: 6208-6218. http://doi:10.1158/1078-0432.CCR$12-0644$

23. Loktev, A. et al. A Tumor-Imaging Method Targeting Cancer-Associated Fibroblasts. J Nucl Med. 2018; 59: 1423-1429. http://doi:10.2967/jnumed.118.210435

24. Giesel, F. L. et al. FAPI-74 PET/CT Using Either (18)F-AlF or Cold-Kit (68)Ga Labeling: Biodistribution, Radiation Dosimetry, and Tumor Delineation in Lung Cancer Patients. J Nucl Med. 2021; 62: 201-207. http://doi:10.2967/jnumed.120.245084

25. Hao, B., Wu, J., Pang, Y., Sun, L. \& Chen, H. 68Ga-FAPI PET/CT in Assessment of Leptomeningeal Metastases in a Patient With Lung Adenocarcinoma. Clin Nucl Med. 2020; 45: 784-786. http://doi:10.1097/RLU.0000000000003231

26. Loutfi, S., Khankan, A. \& Al Ghanim, S. Guidelines for multimodality radiological staging of lung cancer. J Infect Public Health. 2012; 5 Suppl 1: S14-21. http://doi:10.1016/j.jiph.2012.09.009

27. Wood, D. E. et al. Lung Cancer Screening, Version 3.2018, NCCN Clinical Practice Guidelines in Oncology. J Natl Compr Canc Netw. 2018; 16: 412-441. http://doi:10.6004/jnccn.2018.0020

28. Nomori, H. et al. Evaluation of F-18 fluorodeoxyglucose (FDG) PET scanning for pulmonary nodules less than $3 \mathrm{~cm}$ in diameter, with special reference to the CT images. Lung Cancer. 2004; 45: 19-27. http://doi:10.1016/j.lungcan.2004.01.009

29. Chen, H., Pang, Y., Meng, T., Yu, X. \& Sun, L. 18F-FDG and 68Ga-FAPI PET/CT in the Evaluation of Ground-Glass Opacity Nodule. Clin Nucl Med. 2021; 46: 424-426.

http://doi:10.1097/RLU.0000000000003600

30. Wei, Y. et al. [(18)F]AIF-NOTA-FAPI-04 PET/CT uptake in metastatic lesions on PET/CT imaging might distinguish different pathological types of lung cancer. Eur J Nucl Med Mol Imaging. 2021. http://doi:10.1007/s00259-021-05638-z

31. Moreno-Ruiz, P. et al. Stromal FAP is an independent poor prognosis marker in non-small cell lung adenocarcinoma and associated with p53 mutation. Lung Cancer. 2021; 155: 10-19. http://doi:10.1016/j.lungcan.2021.02.028

\section{Figures}




\section{Figure 1}

High level of FAP expression in a SCC tumor (a-c) and low level of FAP expression in a SCLC tumor (d-f) (a) CT image showed a mass in the upper lobe of left lung (black arrow). (b) SCC was diagnosed by pathological examination using HE staining. (c) High FAP level (Score $=6$ ) in tumor was observed on the immunohistochemical analysis. (d) CT image showed a mass in the upper lobe of right lung (black arrow). (e) SCLC was diagnosed by pathological examination using HE staining. (f) Low FAP level (Score $=3$ ) in tumor was observed on the immunohistochemical analysis; scale bar $200 \mu \mathrm{m}$.
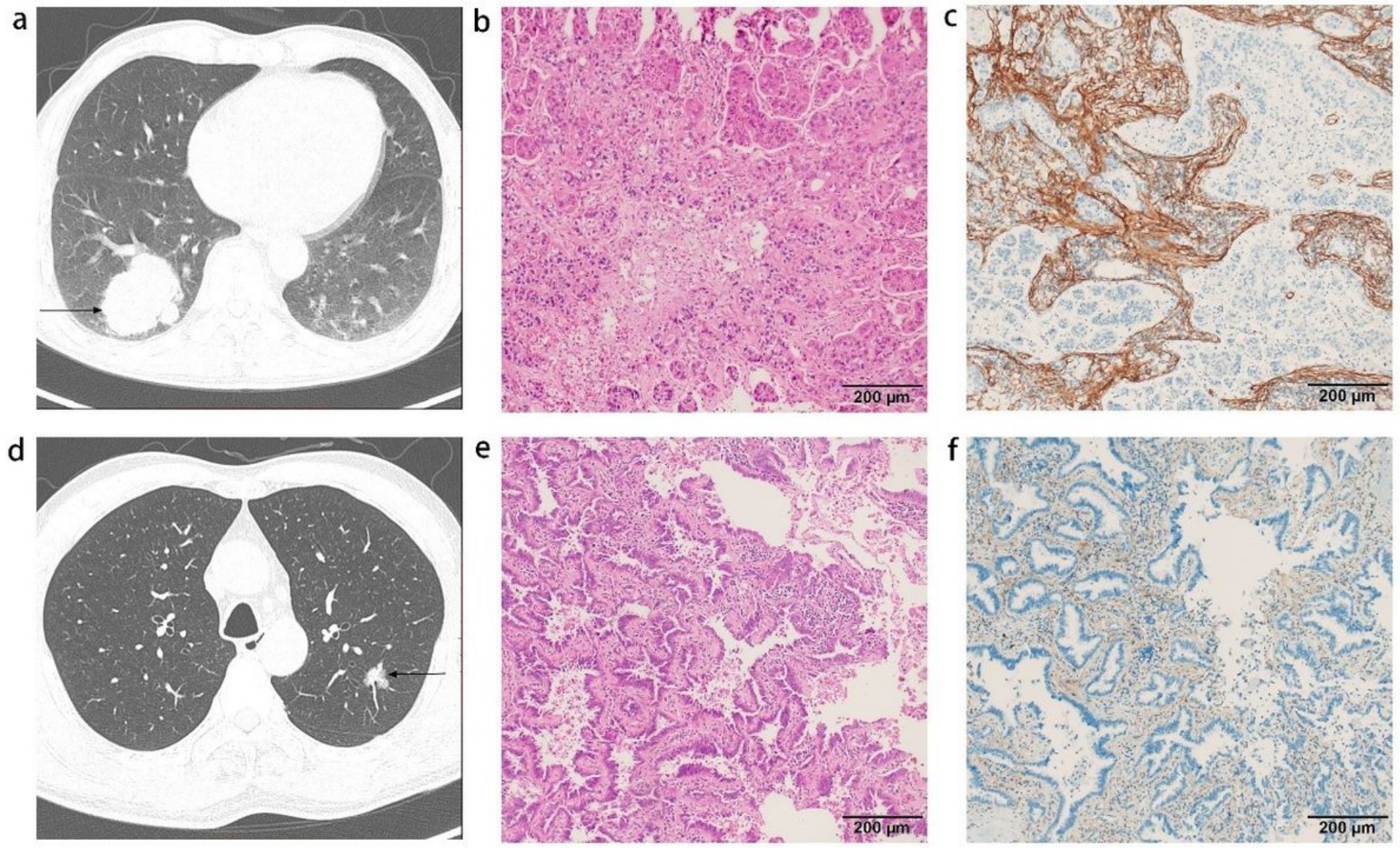

Figure 2

High level of FAP expression in an ADC tumor (a-c) and low level of FAP expression in another ADC tumor (d-f) (a) CT image showed a mass in the lower lobe of right lung (black arrow). (b) ADC was diagnosed by pathological examination using HE staining. (c) High FAP level (Score $=6$ ) in tumor was found on the immunohistochemical analysis. (d) CT image showed a nodule in the upper lobe of the left lung (black arrow). (e) ADC was diagnosed by pathological examination using HE staining. (f) Low FAP level (Score = 2 ) in tumor was found on the immunohistochemical analysis; scale bar $200 \mu \mathrm{m}$. 


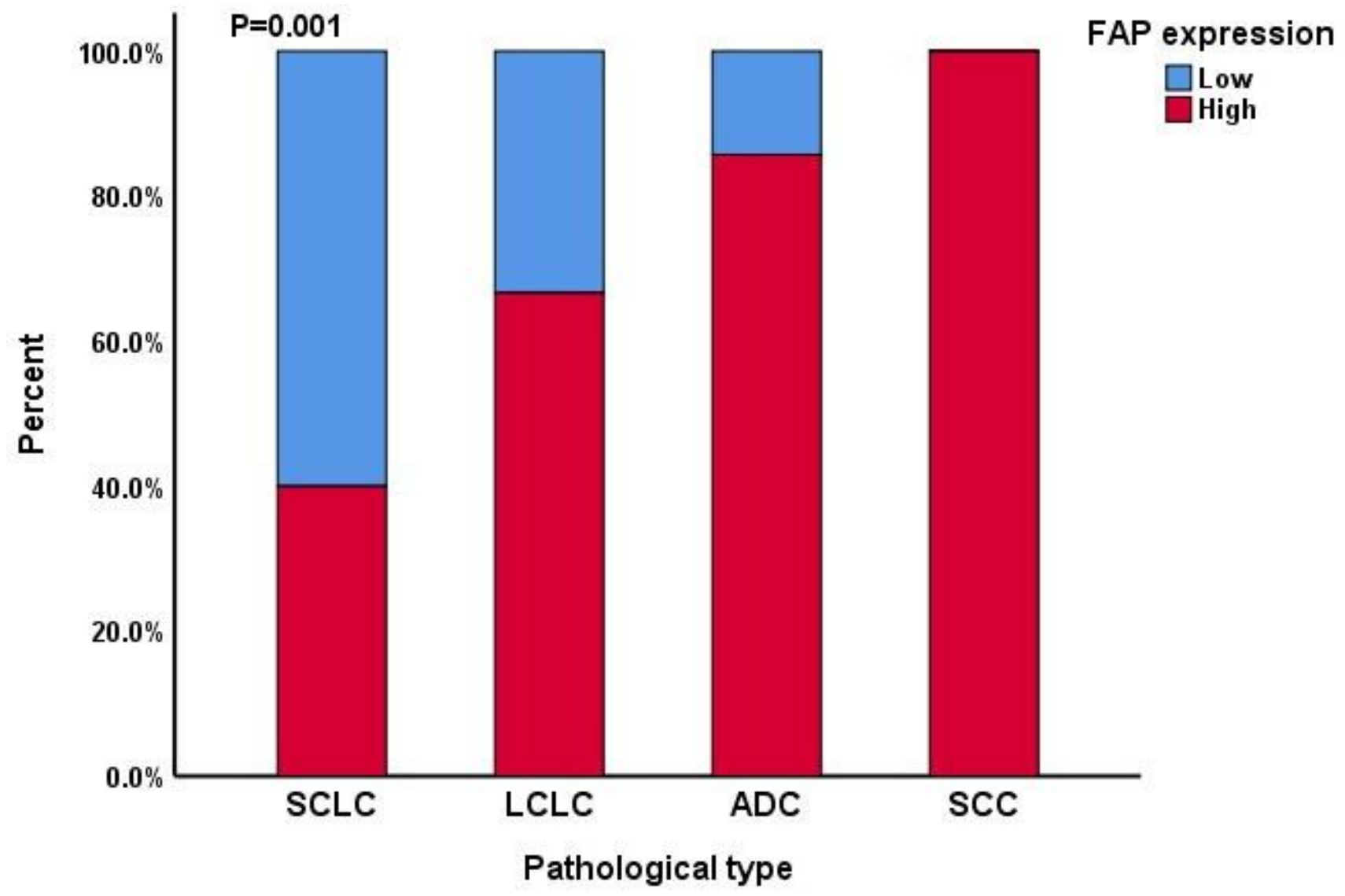

Figure 3

The high and low levels of FAP expression in four different pathological types of LC 
a

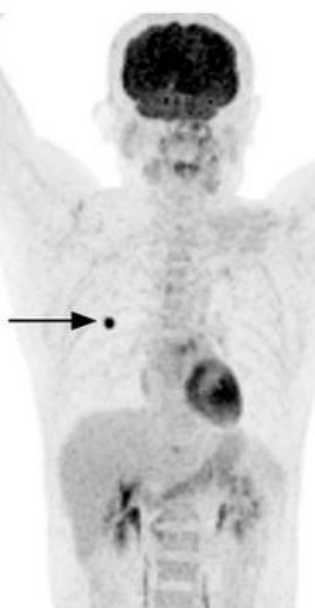

b
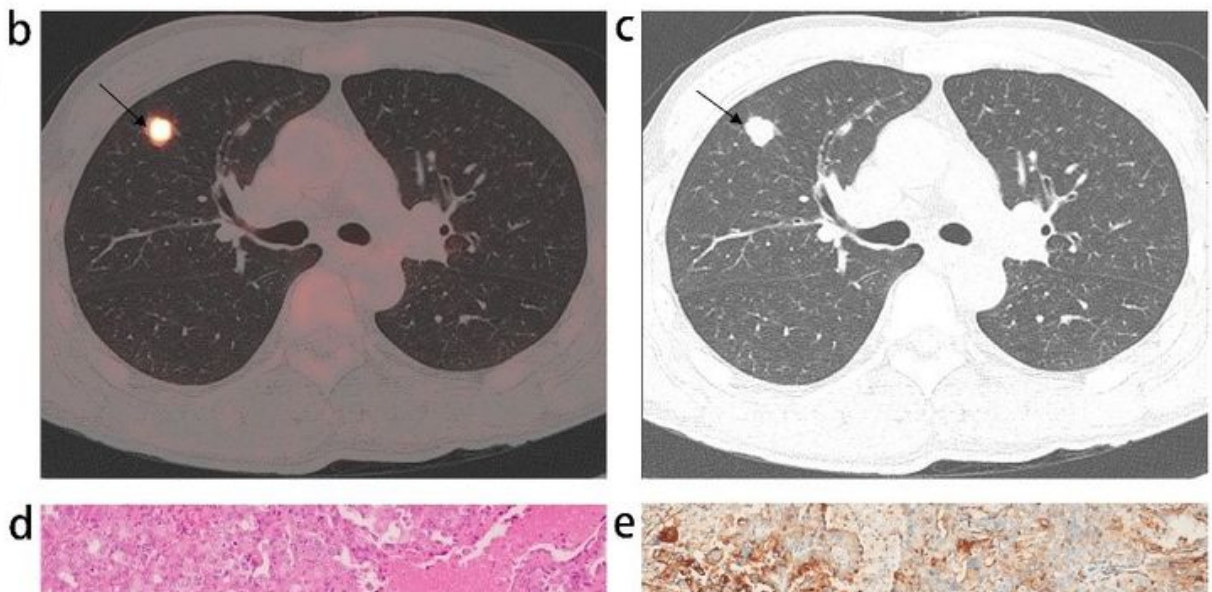

e

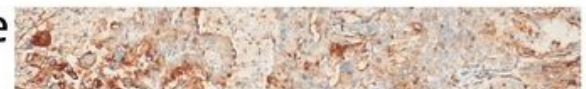

Figure 4

Coincident $\left[{ }^{18} \mathrm{~F}\right]$ F-FDG and FAP expression in a SN tumor of ADC (a-e) and inconsistent $\left[{ }^{18} \mathrm{~F}\right] \mathrm{F}-\mathrm{FDG}$ and FAP expression in a pGGO tumor of ADC (f-j) $(a-c)\left[{ }^{18} F\right]$ F-FDG PET/CT scans showed intense FDG uptake (SUVmax $=12.9$ ) in a SN tumor in middle lobe of the right lung (black arrow in $a, b$ and $c$ ). (d) ADC was diagnosed by pathological examination using HE staining. (e) Consistent with intense $\left[{ }^{18} \mathrm{~F}\right] \mathrm{F}$-FDG uptake, 
high FAP level $($ Score $=6)$ in tumor was also identified by the immunohistochemical analysis. $(f-h)\left[{ }^{18} \mathrm{~F}\right] \mathrm{F}$ FDG PET/CT scans showed no $\left[{ }^{18} \mathrm{~F}\right]$ F-FDG uptake in a pGGO tumor in upper lobe of the right lung (black arrow in $f, g$ and $h$ ). (i) ADC was diagnosed by pathological examination using HE staining. (j) Inconsistent with $\left[{ }^{18} \mathrm{~F}\right]$ F-FDG uptake, positive FAP expression $($ Score $=3$ ) in tumor was found on the immunohistochemical analysis; scale bar $200 \mu \mathrm{m}$.
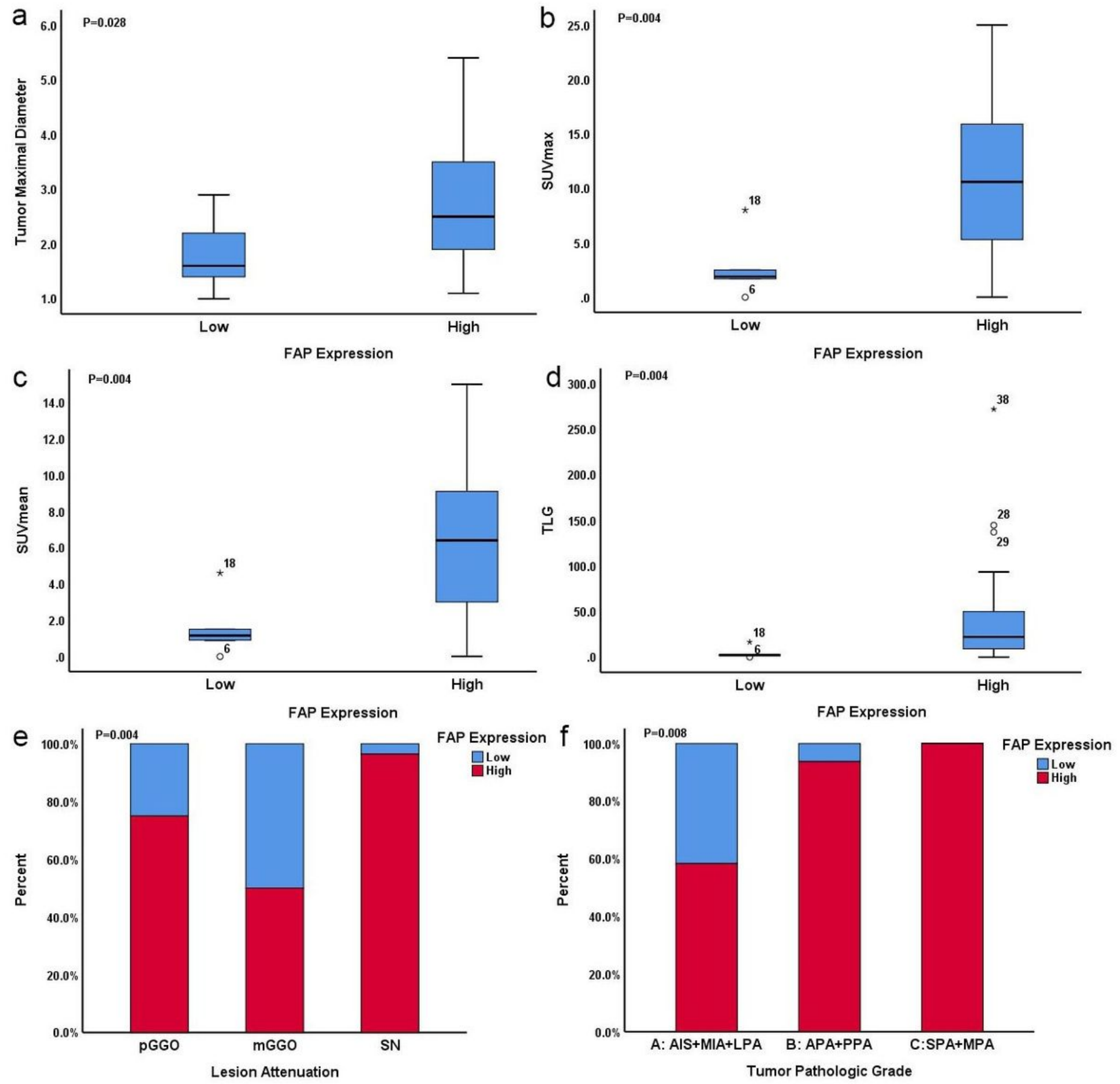

Figure 5 
The relationship of FAP expression in tumor with $\left[{ }^{18} \mathrm{~F}\right]$ F-FDG PET semiquantitative parameters, lesion attenuation and tumor pathologic grade in the patients with non- mucinous ADCs (a) FAP expression level had a close relationship with tumor maximum diameter $(P=0.028)$; (b)FAP expression level had a close relationship with SUVmax $(P=0.003)$; (b) FAP expression level had a close relationship with SUVmean ( $P=0.003)$; (d)FAP expression level had a close relationship with TLG $(P=0.004)$; (e) FAP expression level had a close relationship with tumor attenuation $(P=0.003)$; (f)FAP expression level had a close relationship with tumor pathologic grade $(P=0.005)$. AIS: adenocarcinoma in situ, MIA: minimally invasive adenocarcinoma, LPA: lepidic predominant invasive adenocarcinoma, APA: acinar predominant invasive adenocarcinoma, PPA: papillary predominant invasive adenocarcinoma, SPA: solid predominant invasive adenocarcinoma, MPA: micropapillary predominant invasive adenocarcinoma. 